University of Wollongong

Research Online

Faculty of Engineering and Information

Faculty of Engineering and Information

Sciences - Papers: Part A

Sciences

$1-1-2014$

\title{
Numerical comparison between Berkovich and conical nano-indentations: mechanical behaviour and micro-texture evolution
}

Mao Liu

University of Wollongong, ml818@uowmail.edu.au

Cheng Lu

University of Wollongong, chenglu@uow.edu.au

A Kiet Tieu

University of Wollongong, ktieu@uow.edu.au

Hailiang Yu

University of Wollongong, hailiang@uow.edu.au

Follow this and additional works at: https://ro.uow.edu.au/eispapers

Part of the Engineering Commons, and the Science and Technology Studies Commons

Research Online is the open access institutional repository for the University of Wollongong. For further information contact the UOW Library: research-pubs@uow.edu.au 


\title{
Numerical comparison between Berkovich and conical nano-indentations: mechanical behaviour and micro-texture evolution
}

\author{
Abstract \\ A crystal plasticity finite element method (CPFEM) model has been developed to investigate the influence \\ of indenter geometry on the mechanical behaviour and micro-texture evolution during nano-indentation of \\ single crystal aluminium. The developed model has been validated by comparison with experimental \\ observations. The numerical results show that indenter geometry influences the load-indentation depth \\ curve, hardness and elastic modulus significantly. The surface profile, equivalent plastic strain distribution \\ and micro-texture evolution during nano-indentation have been analysed in detail.

\section{Disciplines} \\ Engineering | Science and Technology Studies

\section{Publication Details} \\ Liu, M., Lu, C., Tieu, K. \& Yu, H. (2014). Numerical comparison between Berkovich and conical nano- \\ indentations: mechanical behaviour and micro-texture evolution. Materials Science and Engineering A: \\ Structural Materials: Properties, Microstructure and Processing, 619 57-65.
}


Numerical comparison between Berkovich and conical nano-indentations: mechanical behaviour and micro-texture evolution

\author{
Mao Liu, Cheng $\mathrm{Lu}^{1}$, Kiet Tieu, Hailiang Yu \\ School of Mechanical, Materials and Mechatronics Engineering, University of Wollongong, \\ Northfields Avenue, Wollongong, NSW 2522, Australia
}

\begin{abstract}
A Crystal Plasticity Finite Element Method (CPFEM) model has been developed to investigate the influence of indenter geometry on the mechanical behaviour and micro-texture evolution during nano-indentation of single crystal aluminium. The developed model has been validated by comparison with experimental observations. The numerical results show that indenter geometry influences the load-indentation depth curve, hardness and elastic modulus significantly. The surface profile, equivalent plastic strain distribution and microtexture evolution during nano-indentation have been analysed in detail.
\end{abstract}

Keywords: Crystal plasticity finite element method; Nano-indentation; Texture; Single crystal aluminium

\title{
1. Introduction
}

Nano-indentation is one of the most popular methods used to investigate the mechanical properties of small-volume bulk materials and thin film materials. A variety of indenter shapes, including pyramidal, conical and spherical, are adopted in nano-indentation. Among them, the Berkovich indenter with a tetrahedral shape is the most frequently used. The popularity of the Berkovich indenter is due to the following features: 1) it has a constant areato-depth ratio that helps yield a hardness value that is independent of the load; 2) it is sharper than most of other indenters and the sharpness enables measurement of the smaller possible testing volume. Conical indenters with various semi-apex angles have also been widely used $[1,2]$, especially in simulations of nano-indentation. A conical indenter with a $70.3^{\circ}$ semiapex angle has the same projected area as the Berkovich indenter. An illustration of the major geometrical parameters of the Berkovich and conical indenters is shown in Fig.1. There is always a concern that different indenter geometries may give different mechanical properties.

\footnotetext{
${ }^{1}$ Corresponding author. Lu C., E-mail: chenglu@,uow.edu.au
} 

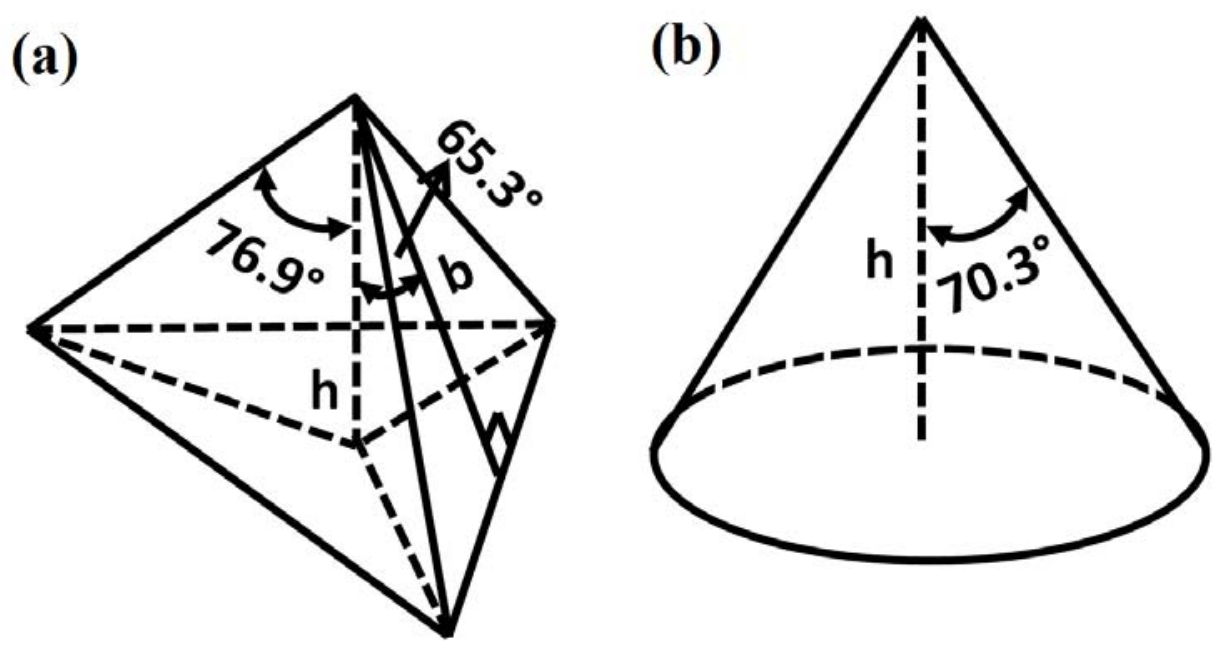

Fig.1. Geometrical parameters of indenters

(a) Berkovich indenter; (b) conical indenter with a $70.3^{\circ}$ semi-apex angle.

Bhattacharya and Nix [3] investigated the indentation process by the classic elasto-plastic Finite Element Method (FEM). In their two-dimensional simulation, a conical indenter with a $68^{\circ}$ semi-apex angle was used to simulate the pyramidal indenter used in the experiment. They found that this did not cause any noticeable difference in the load-indentation depth response of the material. Three-dimensional elasto-plastic FEM simulations of indentation using different indenters have been carried out by Li et al. [2]. It has been found that the load-indentation depth curves of the Berkovich and Vickers indenters were close to those of a conical indenter having a semi-apex angle of $70.3^{\circ}$, despite differences in the stress and strain fields under the indenters. Sakharova et al. [1] carried out three-dimensional numerical simulations of Berkovich, Vickers and conical nano-indentations for both bulk and composite film/substrate materials. For bulk materials with higher ratios of the residual indentation depth after unloading $\left(\mathrm{h}_{\mathrm{f}}\right)$ to the indentation depth at the maximum load $\left(\mathrm{h}_{\max }\right)$, the loadindentation depth curves of the three indenters were very similar. However, Sakharova et al. [1] found that for bulk materials with lower $h_{f} / h_{\max }$ ratios, the Berkovich indenter yielded the highest hardness and the conical indenter had the lowest hardness, while the hardness value obtained by the Vickers indenter lied between the two others. The difference has been attributed to different geometries of the plastic strain regions induced by three types of the indenters. Recently, Kang et al.[4] have numerically investigated the effects of indenter geometry on the load-indentation depth curve, hardness and elastic modulus using the classic elasto-plastic FEM. They found that the Berkovich indenter gave about 25\% higher hardness values than its equivalent conical indenter with a $70.3^{\circ}$ semi-apex angle. Qin et al. [5] also 
reported based on the FEM analysis that difference in hardness measured by the Berkovich indenter and its equivalent conical indenter was about $13 \%$ for copper. The results of both Kang et al. [4] and Qin et al. [5] are in contrast to the conclusions of Bhattacharya and Nix [3] and Li et al. [2]. Swaddiwudhipong et al. [6] used three-dimensional and two-dimensional FEM modeling to study the load-indentation depth response of a wide range of elasto-plastic materials obeying power law strain-hardening for both the Berkovich indenter and its equivalent conical indenter. The results demonstrated that the equivalency between these two indenters in terms of the load-indentation depth curve was not valid across the range of materials properties under study. Shi et al. [7] reported that the widely used equivalence between the Berkovich indenter and its equivalent conical indenter based on equal projected area leaded to significant errors in micro-indentation. The reason is that despite the same projected area, the Berkovich indenter has a more complex shape than the conical indenter, which leads to larger strain and strain gradient, and therefore higher indentation hardness. Shim et al. [8] also found that a significant difference between the two indenters exists for the contact areas and contact stiffnesses.

The classic elasto-plastic FEM have been widely used in the most above studies. In the present study a crystal plasticity finite element method (CPFEM) model will be developed to simulate nano-indentations of the Berkovich and conical indenters. The dependence of hardness and elastic modulus on indenter geometry will be discussed in details. Evolution of micro-texture subject to nano-indentation will be analysed.

\section{Three-dimensional CPFEM simulation model}

The crystal plasticity theory is based on the assumption that plastic deformation is the cumulative effect of crystalline slips in all activated slip systems. Details of the crystal plasticity theory can be found in Ref [9]. The crystal plasticity constitutive model used in the present study follows the approach described by Asaro [10]. It was incorporated into the implicit finite element code ABAQUS/Standard through a User-defined MATerial (UMAT) subroutine. The UMAT subroutine provides the Jacobian matrix for the constitutive model, and updates the stresses and the solution-dependent state variables. In the present study, we adopted the UMAT framework initially developed by Huang [11] and used Bassani and Wu's [12] formulation of the hardening model which has been described in Ref.[13]. The formulations of the rate-dependent hardening model used in the present study can be expressed as [14]: 


$$
\dot{\gamma}^{(\alpha)}=\dot{\gamma}_{0}^{(\alpha)} \operatorname{sgn}\left(\tau^{(\alpha)}\right)\left|\tau^{(\alpha)} / \tau_{\mathrm{c}}^{(\alpha)}\right|^{\mathrm{n}}
$$

where

$$
\operatorname{sgn}(x)=\left\{\begin{array}{r}
-1, x<1 \\
1, x \geq 1
\end{array}\right.
$$

where $\dot{\gamma}_{0}^{(\alpha)}$ is the reference (initial) value of the shear strain rate, which is a constant for all slip systems. $\mathrm{n}$ is the strain rate-sensitive exponent. Both $\dot{\gamma}_{0}^{(\alpha)}$ and $\mathrm{n}$ are material parameters. $\tau_{\mathrm{c}}^{(\alpha)}$ is the critical resolved shear stress of the slip system $\alpha$, which represents the strength of the material.

The rate of change of the critical resolved shear stress is expressed by [15]:

$$
\dot{\tau}_{c}{ }^{(\alpha)}=\sum_{\beta=1}^{N} h_{\alpha \beta} \dot{\gamma}^{(\beta)}
$$

where

$$
\begin{array}{r}
h_{\alpha \alpha}=\left[\left(h_{0}-h_{s}\right) \operatorname{sech}^{2}\left(\left(h_{0}-h_{s}\right) \gamma^{(\alpha)} /\left(\tau_{1}-\tau_{0}\right)\right)+h_{s}\right] \times\left[1+\sum_{\beta=1}^{N} f_{\alpha \beta} \tanh \left(\gamma^{(\beta)} / \gamma_{0}\right)\right] \\
\text { for } \alpha=\beta
\end{array}
$$

and

$$
h_{\alpha \beta}=\mathrm{q} h_{\alpha \alpha}, \quad \text { for } \alpha \neq \beta
$$

here $h_{\alpha \beta}$ is the hardening modulus including the self-hardening of each system $(\alpha=\beta)$ and latent hardening $(\alpha \neq \beta)$; $\mathrm{q}$ is the latent hardening parameter; $\gamma_{0}$ is the reference slip value; $\gamma$ is the shear strain; $\tau_{0}$ is the initial critical resolved shear stress; $\tau_{1}$ is the breakthrough stress corresponding to initiation of large plastic flow; $h_{0}$ is the hardening modulus just after the initial yield; $h_{s}$ is the hardening modulus during easy glide and $f_{\alpha \beta}$ is the magnitude of a particular slip interaction between two slip systems $\alpha$ and $\beta$.

$f_{\alpha \beta}$ depends on the type of dislocation junction formed between slip systems $\alpha$ and $\beta$, which in turn depends on the geometric relation between the two slip systems. Slip 
interactions in FCC crystals can classified into five groups and, consequently, the factors $f_{\alpha \beta}$ are given in terms of five constants, $\left.a_{1}-a_{5}[16]: 1\right) a_{1}$ is associated with the collinear junction between two perfect dislocations of the same Burgers vector gliding on different slip planes; 2) $a_{2}$ is associated with the Hirth lock junction between two perfect dislocations with perpendicular Burgers vectors gliding on intersecting slip planes; 3) $a_{3}$ is associated with the coplanar junction between two perfect dislocations that belong to the same slip plane; 4) $a_{4}$ is associated with the glissile junction between two perfect dislocations gliding on intersecting slip planes, and the resultant Burgers vector is on one of the two slip planes; 5) $a_{5}$ is associated with the sessile (Lomer lock) junction between two perfect dislocations gliding on intersecting slip planes, but the resultant Burgers vector is not on either of the two slip planes.
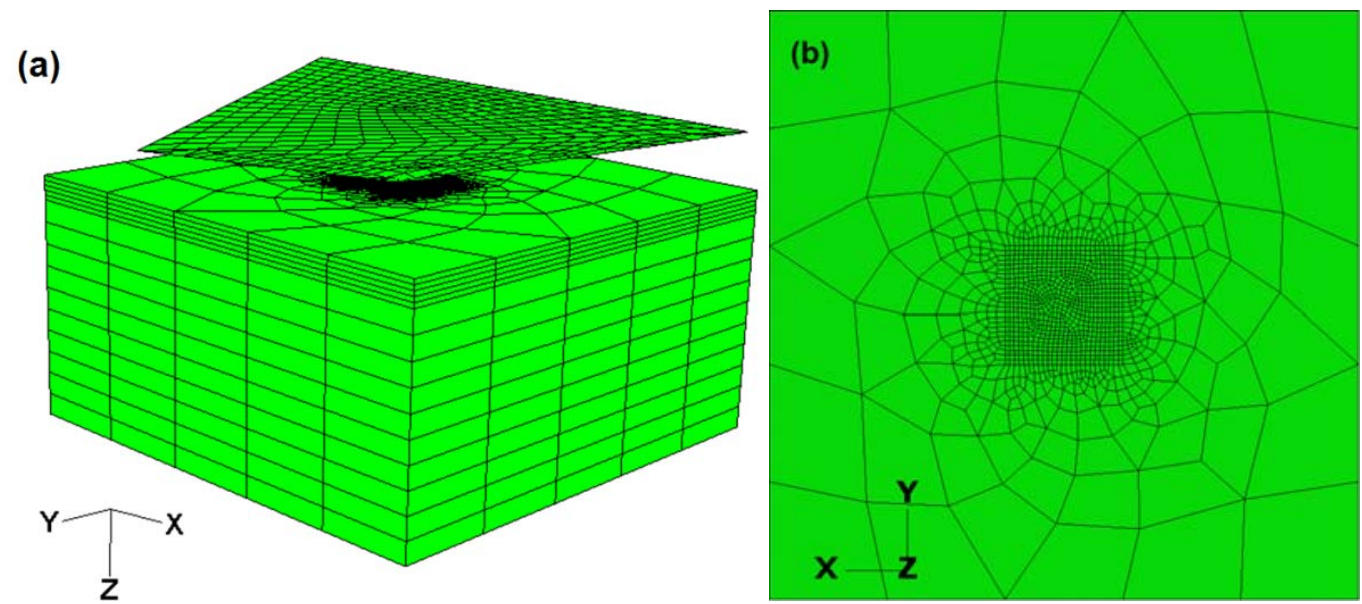

Fig.2 3D Nano-indentation model setup

(a) simulation model using the Berkovich indenter; (b) mesh morphology on the top surface

The computational domain/mesh of the three dimensional nano-indentation model developed in this study is shown in Fig. 2. The Berkovich indenter and the conical indenter $\left(70.3^{\circ}\right.$ semi-apex angle and a $200 \mathrm{~nm}$ radius) have been used and treated as rigid bodies in the simulations. The dimensions of the simulated specimen were $40 \mu \mathrm{m}, 40 \mu \mathrm{m}$ and $20 \mu \mathrm{m}$ and the coordinate system has been marked in Fig. 2. One base edge of the Berkovich indenter was paralleled to the $\mathrm{X}$ axis. The height of the specimen was 20 times the maximum indentation depth $(1 \mu \mathrm{m})$. This satisfies the $10 \%$ rule that the specimen should be at least 10 times thicker than the depth of indentation in order to avoid the influence of the boundary condition [16]. 
The simulated specimen consisted of 17040 eight-node brick elements and 18352 nodes with reduced integration (element id: C3D8R) to ensure that the mesh was fine enough, especially near the contact area with the indenter. The total number of nodes and elements were about 7 times those used in most published papers [17, 18]. In order to compare with the experimental data the simulated specimen was set to be a single crystal aluminium with the same crystallographic orientation as used in the experiment of Ref [19], namely [2 $3 \overline{16}]$, [11 $\overline{2} 1]$ and [ $\overline{1} \overline{6} \overline{1}$ ] along the $X, Y$ and $Z$ axes, respectively.

All the nodes on the bottom surface and the four surrounding surfaces of the simulated specimen were constrained along their normal to the surfaces. The load-indentation depth curve has been found to be independent of the frictional condition [1]. Therefore, a frictionless condition at the indenter/specimen contact interface was adopted in this study. During the simulation the indenter moved down along the $\mathrm{Z}$ direction for about 34000 simulation steps until it reaches the indentation depth of $1000 \mathrm{~nm}$, followed by moving up to release the load.

Franciosi et al. [20] and $\mathrm{Lu}$ et al. [13] reported that the factor $f_{\alpha \beta}$ can be chosen as $\mathrm{a}_{1}=\mathrm{a}_{2}=\mathrm{a}_{3}=1.75, \mathrm{a}_{4}=2$ and $\mathrm{a}_{5}=2.25$ for single crystal aluminium. Three elastic moduli used were $\mathrm{C}_{11}=112 \mathrm{GPa}, \mathrm{C}_{12}=66 \mathrm{GPa}$ and $\mathrm{C}_{44}=28 \mathrm{GPa}$. Table 1 shows the other material parameters used in the hardening model (Eqs. (1)-(4)). These parameters have been validated in the CPFEM simulations of rolling, tensile test and equal-channel angular pressing $[9,13$, $21]$.

Table 1 Parameters used in the work-hardening model.

\begin{tabular}{cccccccc}
\hline $\mathrm{n}$ & $\begin{array}{c}\dot{\gamma}_{0} \\
(1 / \mathrm{s})\end{array}$ & $\begin{array}{c}\mathrm{h}_{0} \\
(\mathrm{MPa})\end{array}$ & $\begin{array}{c}\mathrm{h}_{s} \\
(\mathrm{MPa})\end{array}$ & $\begin{array}{c}\tau_{1} \\
(\mathrm{MPa})\end{array}$ & $\begin{array}{c}\tau_{0} \\
(\mathrm{MPa})\end{array}$ & $\gamma_{0}$ & $\mathrm{q}$ \\
\hline 300 & 0.0001 & 100 & 0.01 & 6.3 & 6 & 0.001 & 1 \\
\hline
\end{tabular}

\section{Results and discussion}

Kuo and Huang [19] preformed nano-indentation experiments on a single crystal aluminium using a Berkovich indenter. The crystallographic orientation of their specimen is identical to the one used in the present simulation. Fig. 3 compares the experimental loadindentation depth (P-h) curve reported in Ref. [19] and the simulated result using the Berkovich indenter. It can be seen that the simulated curve is in reasonable agreement with the experimental one. At smaller indentation depths the simulated load is higher than the 
measured load. The discrepancy has been reduced as the indentation depth increases. It is noteworthy that the material parameters used in the CPFEM simulation were obtained by matching the experimental results of plane strain compressions in our previous studies [22, 23]. We did not tune any material parameter to fit the measured load-indentation depth curve in this study. There is a gap between two unloading curves at the maximum indentation depth. This is due to the fact that the indenter has been remained at the maximum indentation depth for a period of time in the experiment, while the load was immediately released once the maximum indentation depth was reached in the simulation.

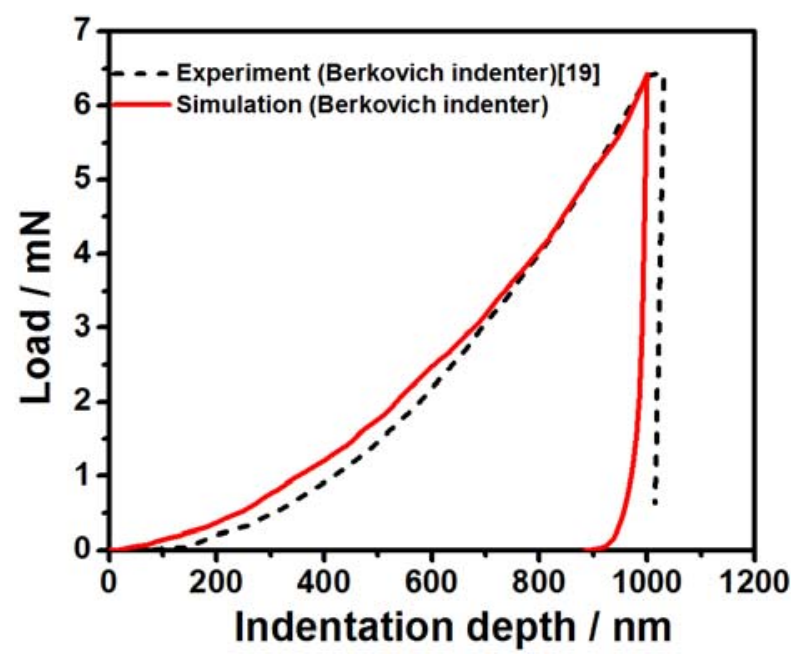

Fig. 3 Comparison of the load-indentation depth curves between experiment and simulation.

A comparison of the load-indentation depth curves for the Berkovich indenter and the conical indenter is shown in Fig. 4. It is obvious that indenter geometry influences the loadindentation depth curve. The load-indentation depth curve of the Berkovich indenter is higher than that of the conical indenter. The indentation hardness $(\mathrm{H})$ was calculated by [24]

$$
\begin{gathered}
H=\frac{P}{24.56 h_{c}{ }^{2}} \\
h_{c}=h-k \frac{P}{\frac{d P}{d h}}
\end{gathered}
$$

where $\mathrm{P}$ is indentation load at the maximum load and $\mathrm{h}$ is the indentation depth at the maximum load. $\mathrm{h}_{\mathrm{c}}$ represents the contact depth. The factor $k$ depends upon the indenter. For the Berkovich indenter $k$ is equal to 0.75 , while $k=0.72$ is for the conical indenter. $\mathrm{dp} / \mathrm{dh}$ is the slope of the unloading curve. The elastic modulus (E) was determined by [24]

$$
E=\frac{1}{\beta} \sqrt{\frac{\pi}{24.5}} \frac{1}{2 h\left(1-v^{2}\right)} \frac{d P}{d h}
$$


where $\beta$ is a constant which is equal to 1.0 and 1.034 for the conical indenter and the Berkovich indenter, respectively. $v$ is the Poisson's ratio of the specimen. $v=0.347$ was used in this study.

The calculated indentation hardness and elastic modulus for the Berkovich indenter are $0.263 \mathrm{GPa}$ and $69.17 \mathrm{GPa}$ respectively. The calculated indentation hardness and elastic modulus for the conical indenter are $0.244 \mathrm{GPa}$ and $77.20 \mathrm{GPa}$ respectively. It is clear that hardness estimated by the Berkovich indenter is higher than that by the conical indenter. The elastic modulus estimated by the Berkovich indenter is close to the standard value of Young's Modulus (70.4 GPa) for aluminium [25], while the conical indenter gives a higher elastic modulus value.

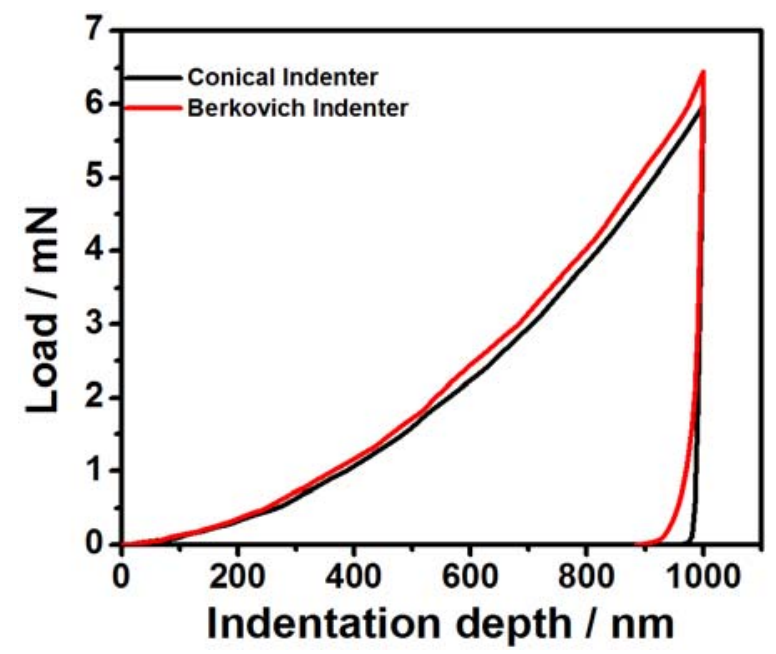

Fig. 4 Comparison of the load-indentation depth curves of the Berkovich indenter and the conical indenter.
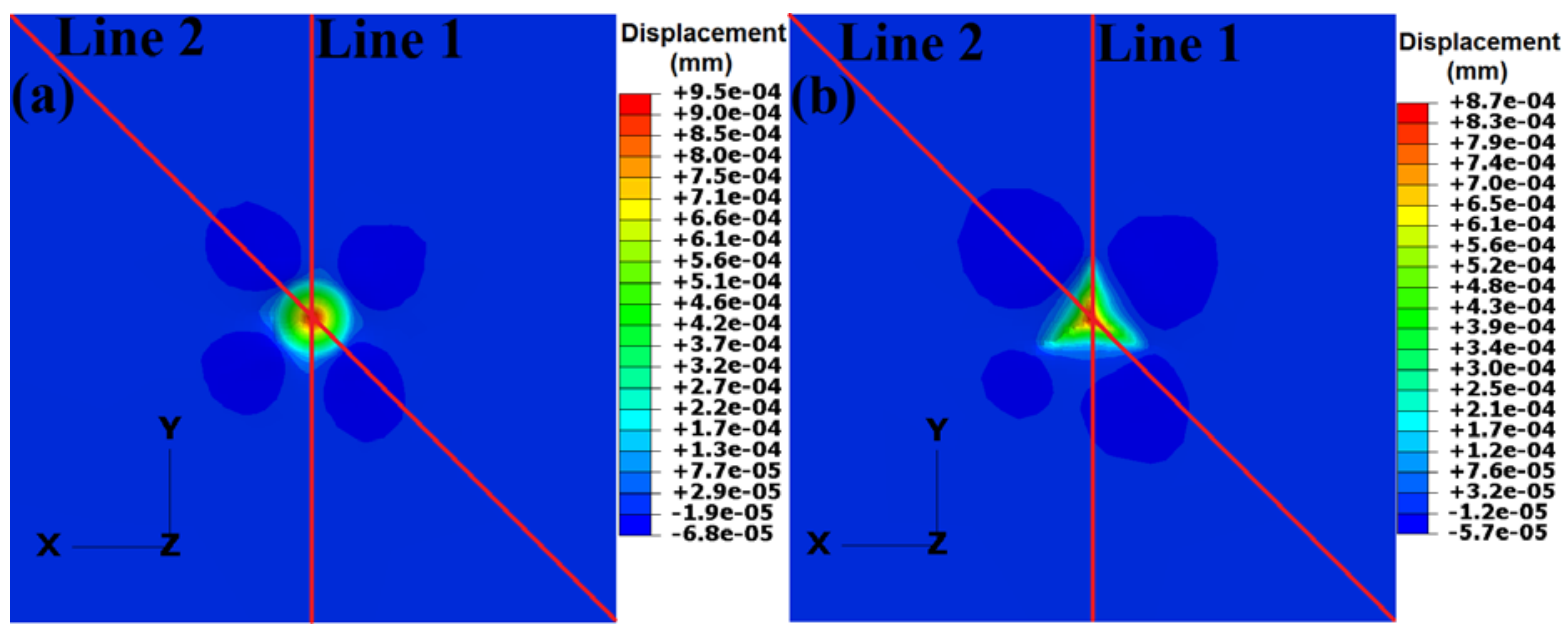

Fig. 5 Simulated contour plots of the out-of-plane displacement for two indenters

(a) conical indenter; (b) Berkovich indenter. 
Fig. 5 compares the simulated contour plots of the out-of-plane displacement on the top surfaces. A twofold symmetry in the out-of-plane displacement can be observed in Fig. 5(a). Fig. 5(b) looks similar to Fig. 5(a) even though the pattern in Fig. 5(b) is not perfectly symmetrical. It can be seen that indenter geometry does not affect the pattern of the out-ofplane profile significantly. Peralta et al. [26] also found that the sinking-in and piling-up behaviour depend on in-plane crystallographic orientations rather than the orientation of the indenter.

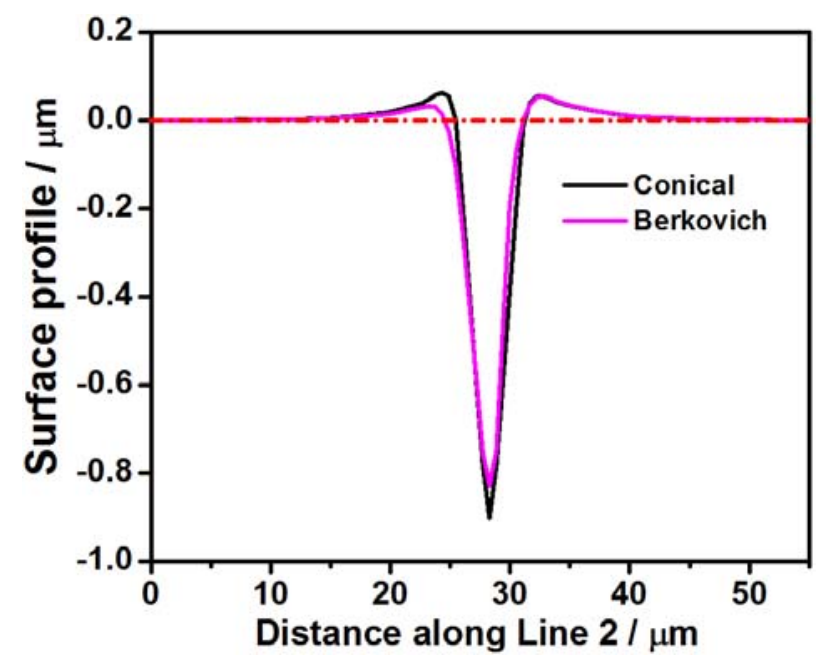

Fig. 6 Comparisons of the surface profile along Line 2 for the two indenters.

Two lines, named Line 1 and Line 2, are marked in Fig. 5. Fig.6 compares the simulated surface profiles of two indenters along Line 2 after unloading. The result indicates that the piling-up is almost symmetrical for the conical indenter. However, the piling-up generated by the Berkovich indenter is unsymmetrical. The piling-up of the Berkovich indenter at the lefthand side of the indent is lower than that of the conical indenter. This suggests that although indenter geometry does not affect the piling-up pattern significantly, it does change the magnitude of the piling-up.

Figs 7(a) and 7(b) show the distributions of the equivalent plastic strain at the maximum indentation depth on a through-thickness cross section passing through Line 1, which is called CS1 in the following context. The maximum strain is located near the left-hand side of the indent and close to the indent tip for the Berkovich indenter, while it appears in the conical indenter case on both sides of the indent and also beneath the indent tip. The maximum value of the equivalent plastic strain is higher for the Berkovich indenter than for the conical indenter. The equivalent plastic strain distribution is shallower and less 'spherical' for the Berkovich indenter than for the conical one, as seen in Fig. 7(a) and Fig. 7(b). These observations suggest that the presence of sharp edges in indenter geometry can influence the 
plastic strain distribution in the indented specimen, which is consistent with the prediction by Sakharova [1].

Figs. 7(c) and 7(d) demonstrate the distributions of the equivalent plastic strain on a through-thickness cross section passing through Line 2, which is called CS2 in the following context. The maximum strain is located on the right-hand side of the indent for the Berkovich indenter. For the conical indenter case, it appears beneath the indenter tip and expands to both sides of the indent tip. In general, the shape of the plastic deformation zone generated by Berkovich indenter is different to that generated by the conical indenter. That is the reason why the load and hardness estimated by Berkovich indenter are different to those by the conical one.
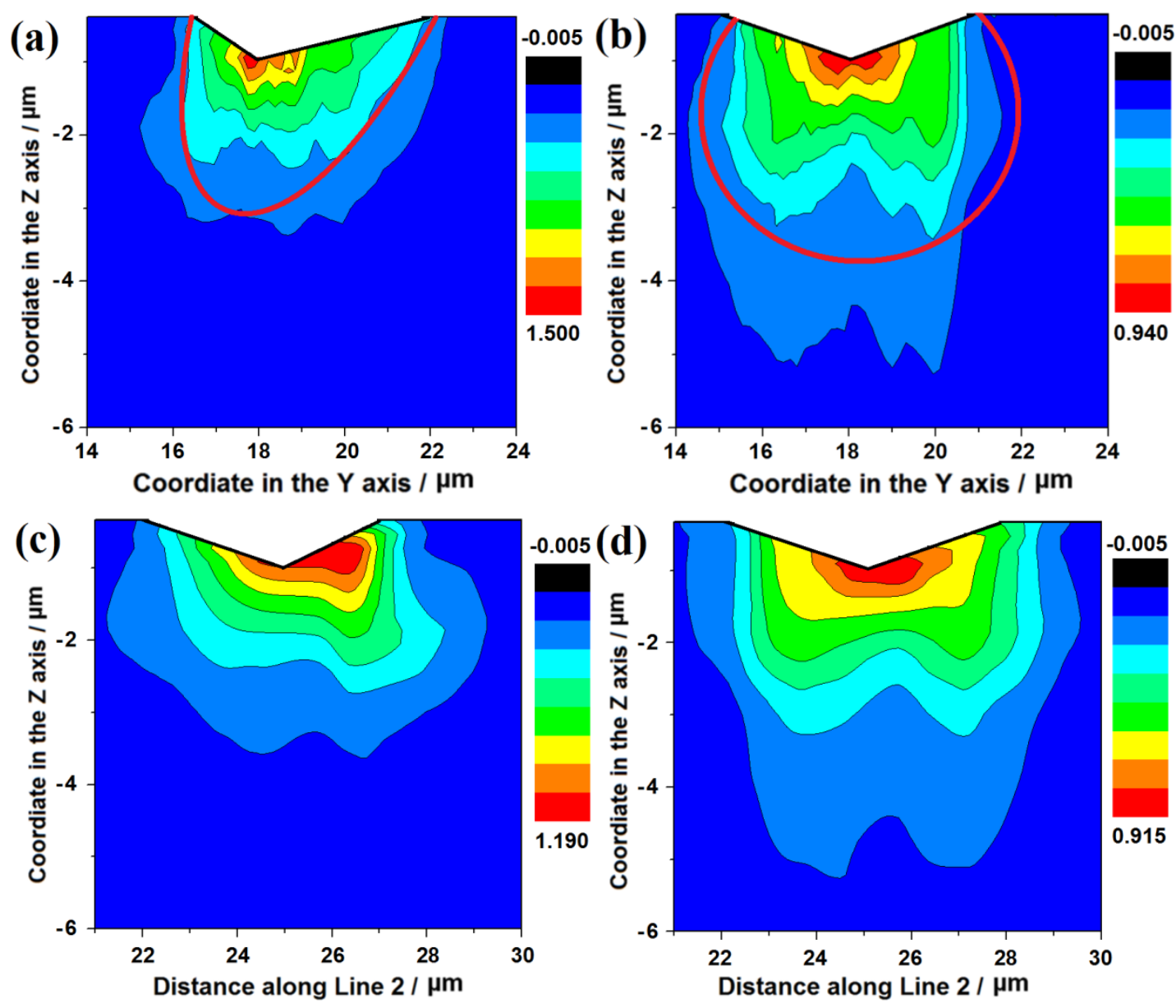

Fig. 7 Distributions of the equivalent strain at the maximum indentation depth:

(a) along Line 1 for the Berkovich indenter; (b) along Line 1 for the conical indenter; (c) along Line 2 for the Berkovich indenter; (d) along Line 2 for the conical indenter.

To analyse evolution in the crystallographic orientation during the indentation process, misorientation of each node relative to its initial orientation was partitioned into three 
components representing the lattice rotation angles around the $\mathrm{X}, \mathrm{Y}$ and $\mathrm{Z}$ axes, respectively, using the method proposed by Wert et al. [27]. Contour maps of the lattice rotation angles around the $\mathrm{X}, \mathrm{Y}$ and $\mathrm{Z}$ axes $\left(\theta_{\mathrm{X}}, \theta_{\mathrm{Y}}\right.$ and $\left.\theta_{\mathrm{Z}}\right)$ on $\mathrm{CS} 1$ are shown in Fig. 8 for the Berkovich indenter. Positive values and negative values represent counter-clockwise rotation and clockwise rotation, respectively. It can be seen in Fig. 8 that the lattice rotations mainly occur near the side surfaces of the indent. Fig. 8(a) shows that large counter-clockwise rotation around the $\mathrm{X}$ axis $\left(\theta_{\mathrm{X}}\right)$ dominates near the right-hand side surface of the indent, while $\theta_{\mathrm{X}}$ is very small near another side surface of the indent except for the region close to the indent tip. The lattice rotation angle around the $\mathrm{Y}$ axis $\left(\theta_{\mathrm{Y}}\right)$ has different signs near two side surfaces of the indent as shown in Fig. 8(b). It is noted from Fig. 8(b) that the lattice has rotated in the clockwise sense to about $10^{\circ}$ near the left-hand side surface of the indent. As observed in Fig. 8 (c) the counter-clockwise rotation around the $\mathrm{Z}$ axis dominates around the indent.
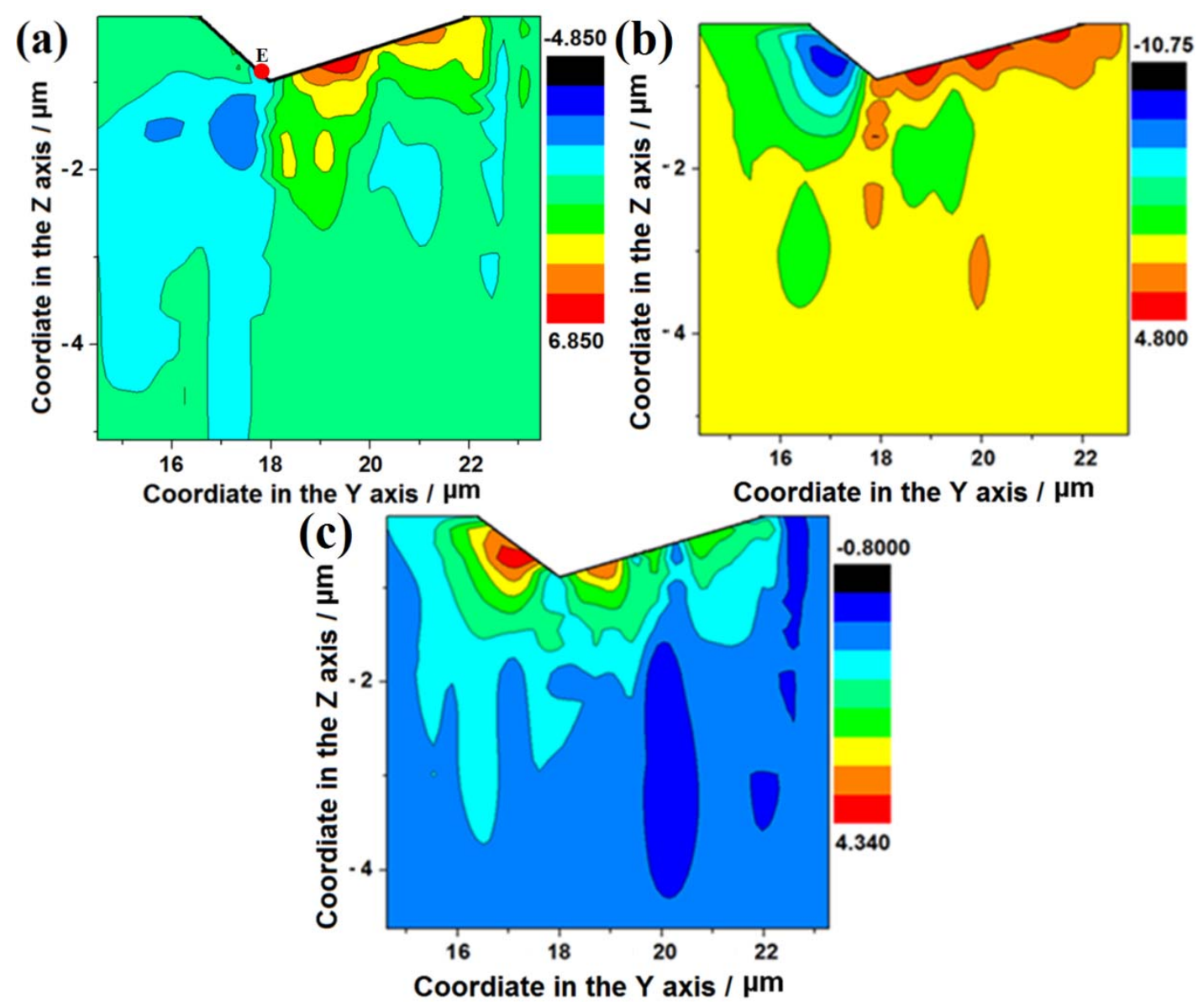

Fig. 8 Contour maps of the lattice rotation angles on CS1 for the Berkovich indenter (a) around the $\mathrm{X}$ axis; (b) around the $\mathrm{Y}$ axis; (c) around the $\mathrm{Z}$ axis. 
Fig 9 shows the contour maps of the lattice rotation angles around the $\mathrm{X}, \mathrm{Y}$ and $\mathrm{Z}$ axes on CS1 for the conical indenter. It can be seen from Fig. 9 that both clockwise and counter clockwise rotations around three directions exist. This is consistent with the observations in Ref. $[28,29]$ which reported the lattice rotates along different directions on two side surfaces of the indent made by a conical indenter. However, it is clear that the patterns of the lattice rotation angle observed in Fig. 9 are different to those in Fig. 8, which indicates that indenter geometry significantly influences micro-texture evolution during the indentation process.
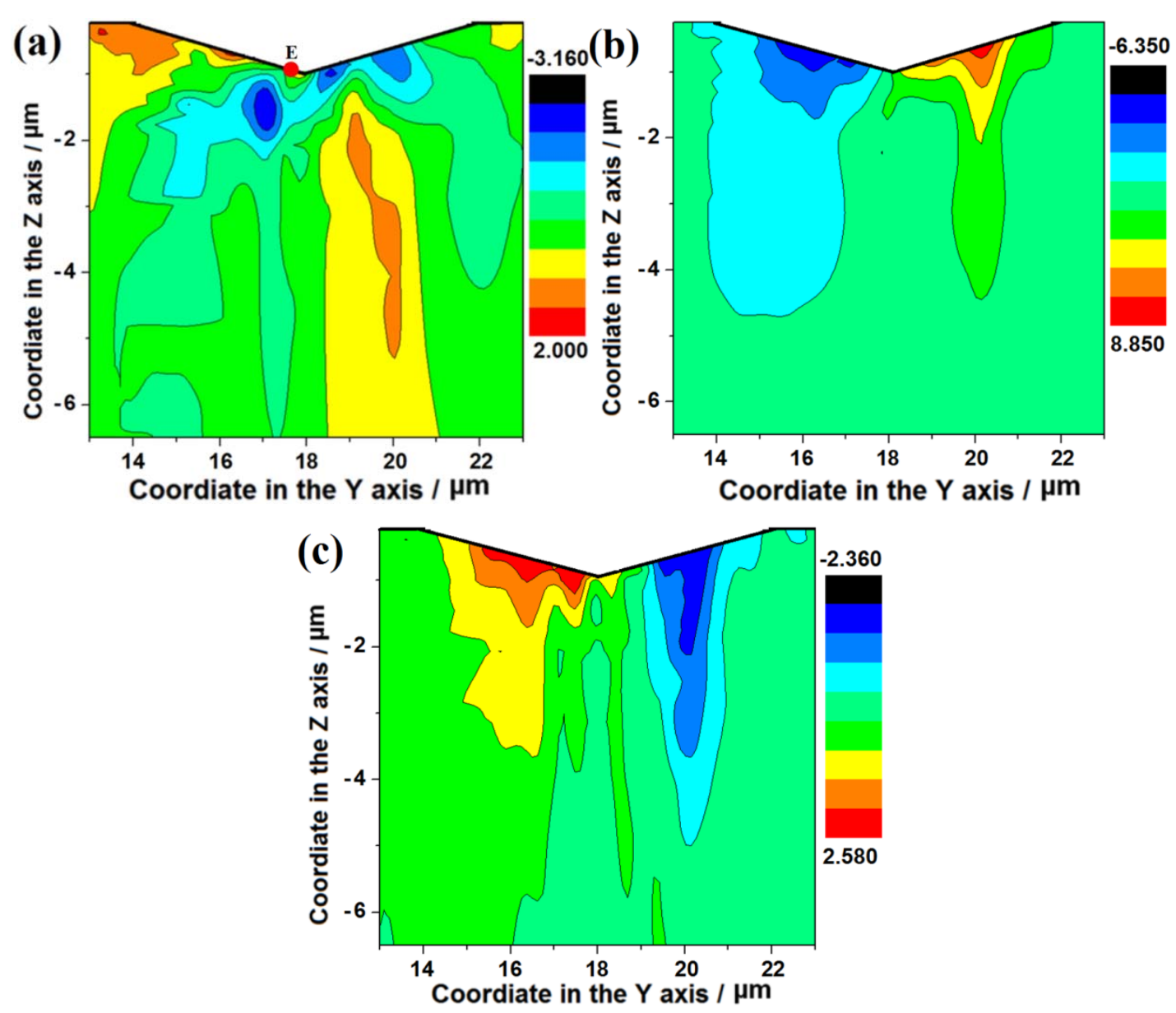

Fig. 9 Contour maps of the lattice rotation angles on CS1 for the conical indenter (a) around the $\mathrm{X}$ axis; (b) around the $\mathrm{Y}$ axis; (c) around the $\mathrm{Z}$ axis.

Fig. 10 shows the contour maps of the lattice rotation angles around the $\mathrm{X}, \mathrm{Y}$ and $\mathrm{Z}$ axes on CS2 of the specimen indented by the Berkovich indenter. It is found in Fig. 10(a) that there are two large lattice rotation regions located beneath the indent tip and with opposition signs. Unlike Fig. 8(a) the largest rotation angle around the $\mathrm{X}$ axis $\left(\theta_{\mathrm{X}}\right)$ is not located on the side surface of the indent in Fig. 10(a). Fig. 10(b) displays that the largest negative $\theta_{\mathrm{Y}}$ exists on the left-hand side surface of the indent. The negative $\theta_{\mathrm{Y}}$ expands from the left-hand side 
surface of the indent to the region below the indent tip. The largest positive $\theta_{\mathrm{Y}}$ has been found in the piling-up region at the left-hand side of the indent. Fig. 10(c) shows that the largest positive and negative $\theta_{Z}$ values appear near the left-hand side surface of the indent and the right-hand piling-up region, respectively.
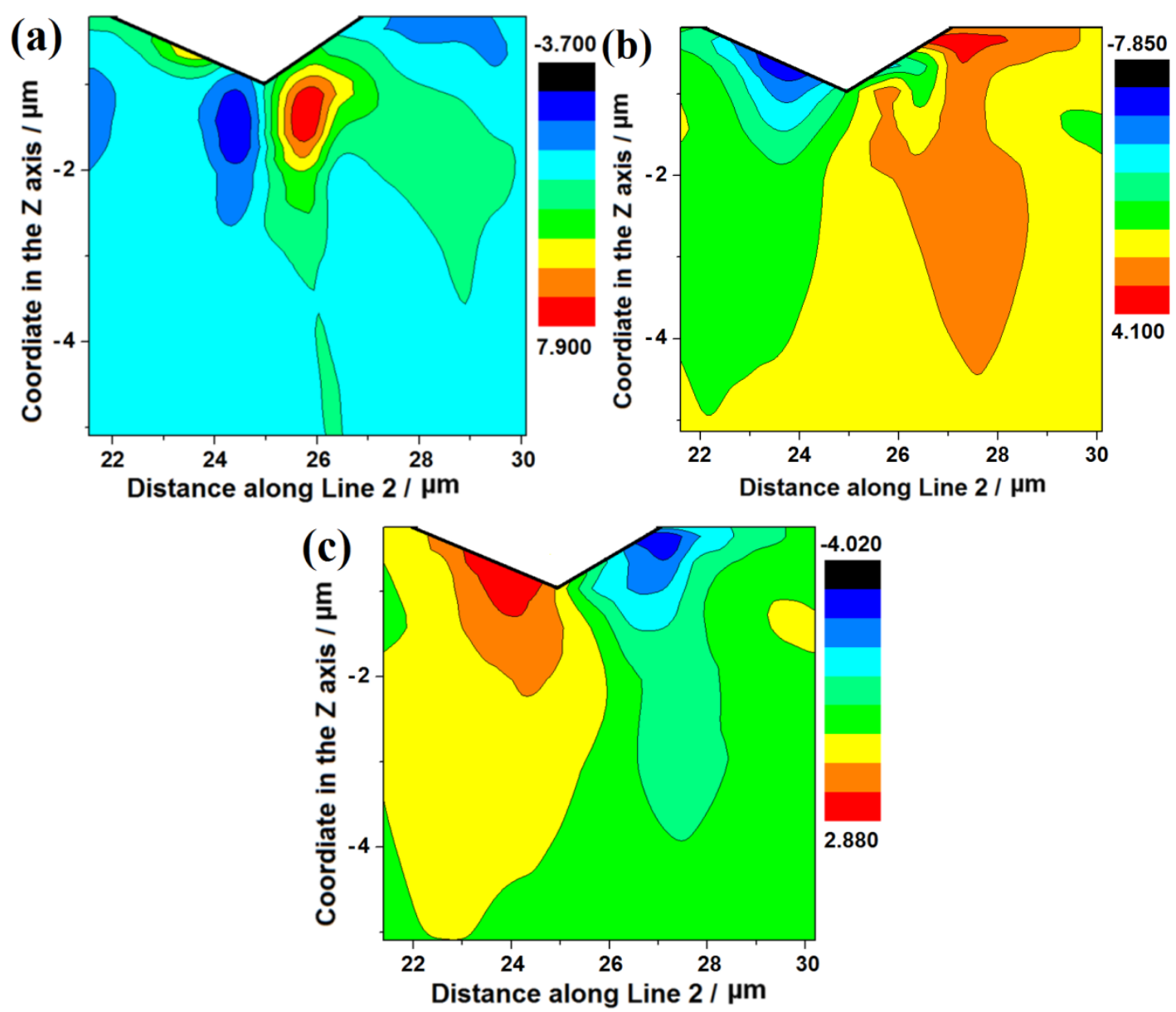

Fig. 10 Contour maps of the lattice rotation angles on CS2 for the Berkovich indenter (a) around the $\mathrm{X}$ axis; (b) around the $\mathrm{Y}$ axis; (c) around the $\mathrm{Z}$ axis.

Fig. 11 shows the contour maps of the lattice rotation angles around the $\mathrm{X}, \mathrm{Y}$ and $\mathrm{Z}$ axes on CS2 for the conical indenter. The patterns of the rotation angles in Fig. 11(b) and Fig. 11(c) are similar to those observed in Fig. 9(b) and Fig. 9(c), respectively. This is due to the symmetrical shape of the conical indenter. However, careful inspection indicates that the pattern in Fig. 11(a) is different to that in Fig. 9(a). The reason can be attributed to different initial lattice orientations relative to the indentation direction in two figures. Comparison between Fig. 10 and Fig. 11 clearly depicts that the patterns of the rotation angles are different for the Berkovich indenter and the conical indenter. This suggests again that the 
geometry of the indenter profoundly affects the rotation of the lattice orientation induced by nano-indentation.
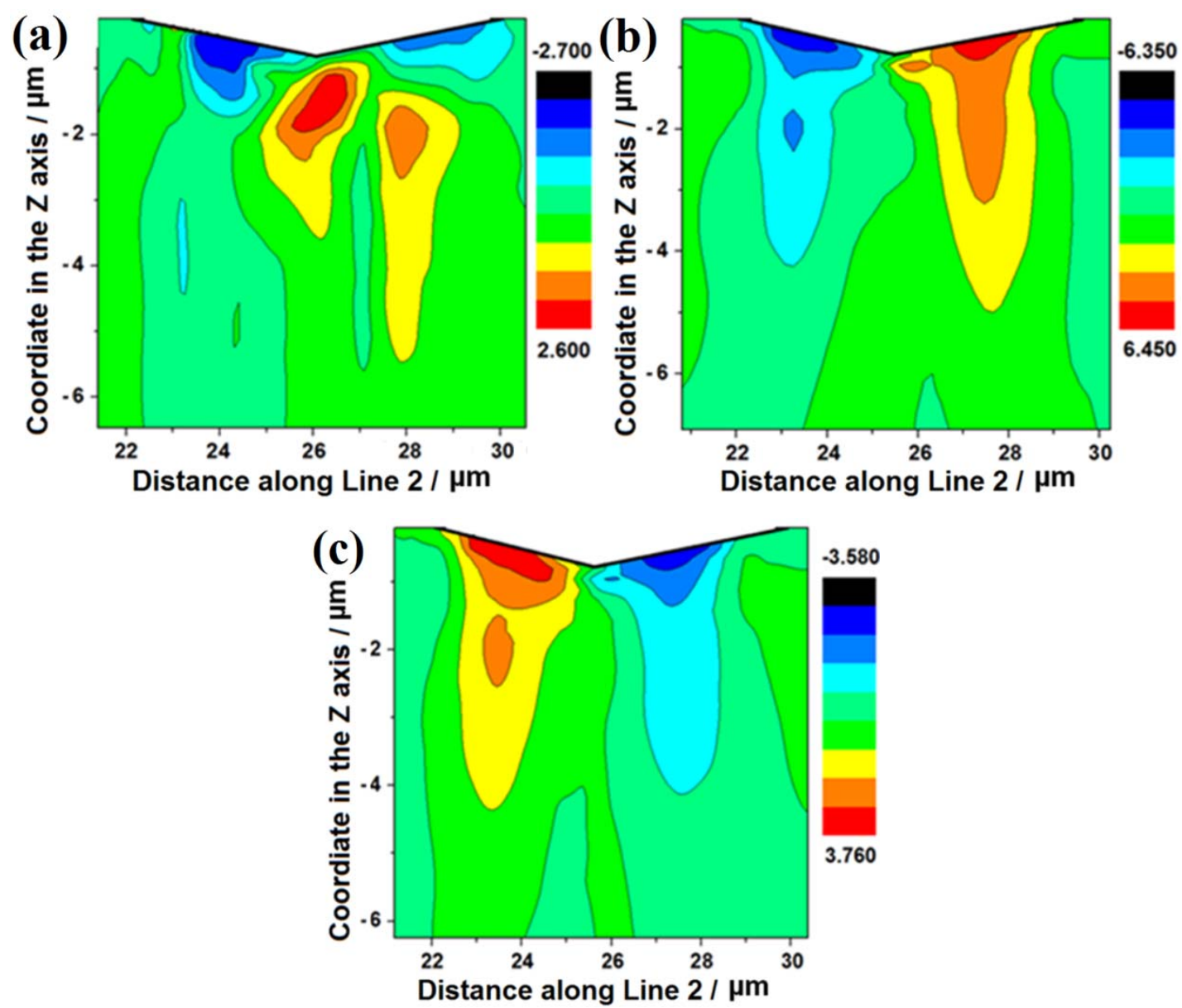

Fig. 11 Contour maps of the lattice rotation angles on CS2 for the conical indenter (a) around the $\mathrm{X}$ axis; (b) around the $\mathrm{Y}$ axis; (c) around the $\mathrm{Z}$ axis.

The differences in the rotation angle between the Berkovich indenter and the conical indenter indicates that different slip systems are activated by the two indenters during nanoindenation. In order to confirm this, an element marked in Fig.8a and Fig.9a with a red dot and a capital letter $E$ has been selected for analysis. Fig. 12 shows the shear strain rate $(\dot{\gamma})$ for all the 12 slip systems of this element. For the Berkovich indentation case, four slip systems $((11-1)[011],(1-11)[10-1],(-111)[101]$ and (11-1)[101]) are active during the early stage of indentation with the indentation depth of less than $200 \mathrm{~nm}$. As the indentation depth increases the slip systems (11-1)[101] and (111)[-110] become the dominant active slip systems. For the conical indentation case more slip systems are active at the early stage of indentation. The slip system (111)[0-11] and (-111)[110] have the positive $\dot{\gamma}$, whereas other six slip systems ((111)[10-1], (1-11)[011], (1-11)[10-1], (11-1)[011], (-111)[0-11] and (11-1)[-110]) have the negative $\dot{\gamma}$. As the indentation depth increases four slip systems ((111)[0-11], (1-11)[011], 
(111)[-110] and (11-1)[101]) become the major active slip systems. It is clear that different slip systems could be activated by different types of the indenters. This is the reason why different distributions of the equivalent plastic strain and lattice rotation angles have been observed for two different indenters and in turn why the load-displacement curve, elastic modulus and hardness measured by the two indenters are not the same.

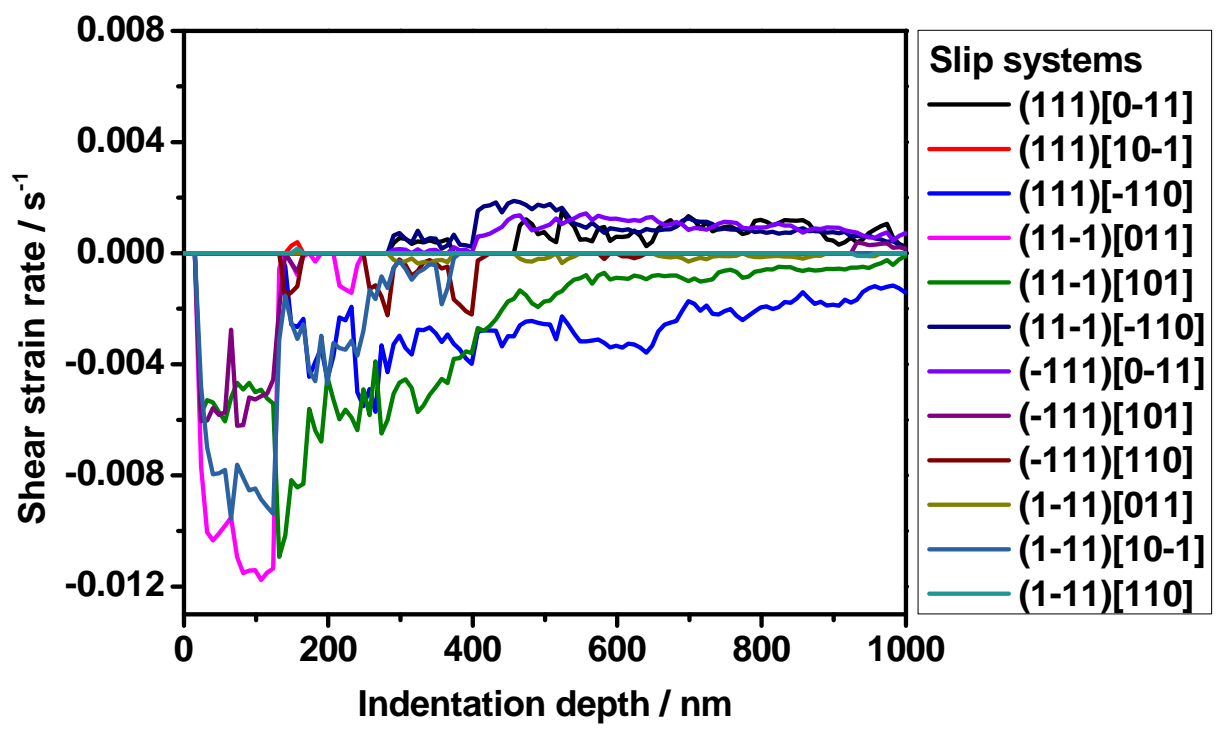

(a)

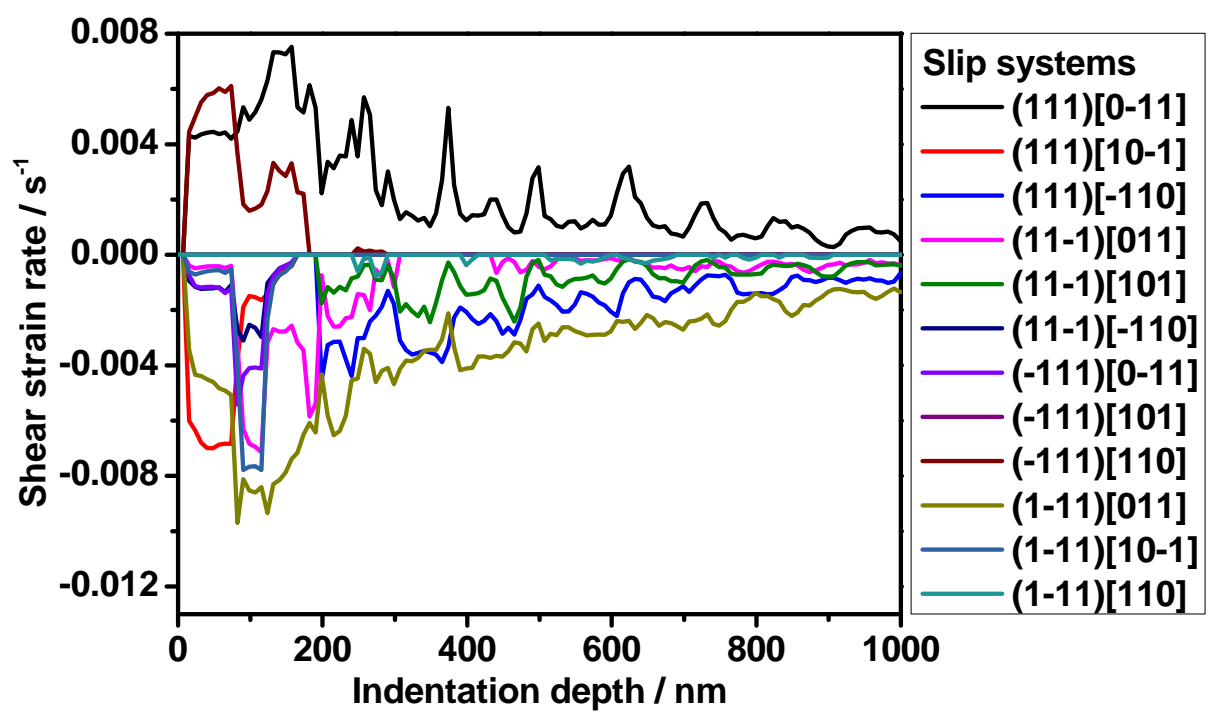

(b)

Fig.12 Shear strain rates of all the 12 slip systems for a selected element

(a) Berkovich indenter; (b) conical indenter 


\section{Conclusions}

1) A crystal plasticity FEM model has been developed to simulate the nano-indentation process. The simulated load-displacement curve has been validated by comparison with experimental results.

2) The simulated load-indentation depth curves have been compared for two different indenters: Berkovich indenter and conical indenter. It has been found that the two simulated curves are different and the Berkovich indenter has higher hardness and lower elastic modulus than the conical indenter. It has also been seen that the pilingup patterns only depend on the in-plane crystallographic orientations rather than the geometry of the indenter. However, the geometry of the indenter does affect the magnitude of the piling-up.

3) The equivalent plastic strain has been analysed on two through-thickness cross sections. It has been found that the deformation zone is smaller for the Berkovich indenter than for the conical one. The location and the magnitude of the maximum equivalent strain are different for the two indenters.

4) A detailed analysis has been carried out to investigate the rotation of the lattice orientation during the indentation process. It has been found that indenter geometry significantly affects the distributions of the lattice rotation angles around the indent.

5) The differences in the equivalent plastic strain distribution and the lattice rotation angle distribution between the two indenters are considered to be the main reasons to cause difference in mechanical behaviour of the specimens indented by two different indenters.

\section{Acknowledgements}

The authors acknowledge the financial support from an Australian Research Council Discovery Grant (DP0773329) and from UPA and IPTA scholarships from the University of Wollongong.

\section{References}


1. Sakharova, N.A., et al., Comparison between Berkovich, Vickers and conical indentation tests: A three-dimensional numerical simulation study. International Journal of Solids and Structures, 2009. 46(5): p. 1095-1104.

2. Li, M., et al., A numerical study of indentation using indenters of different geometry. Journal of Materials Research, 2004. 19(1): p. 73-78.

3. Bhattacharya, A.K. and W.D. Nix, Finite-Element Analysis of Cone Indentation. International Journal of Solids and Structures, 1991. 27(8): p. 1047-1058.

4. Kang, J.J., A.A. Becker, and W. Sun, Effect of indenter geometries on material properties: A Finite Element simulation. Advances in Experimental Mechanics Viii, 2011. 70: p. 219-224.

5. Qin, J., et al., The equivalence of axisymmetric indentation model for three-dimensional indentation hardness. Journal of Materials Research, 2009. 24(3): p. 776-783.

6. Swaddiwudhipong, S., et al., Equivalency of Berkovich and conical load-indentation curves. Modelling and Simulation in Materials Science and Engineering, 2006. 14(1): p. 71-82.

7. Shi, Z., et al., The equivalent axisymmetric model for Berkovich indenters in power-law hardening materials. International Journal of Plasticity, 2010. 26(1): p. 141-148.

8. Shim, S., W.C. Oliver, and G.M. Pharr, A comparison of 3D finite element simulations for Berkovich and conical indentation of fused silica. International Journal of Surface Science and Engineering, 2007. 1(2-3): p. 259-273.

9. $\mathrm{Si}$, L.Y., et al., Simulation of rolling behaviour of cubic oriented al single crystal with crystal plasticity FEM. Journal of Materials Processing Technology, 2008. 201(1-3): p. 79-84.

10. Asaro, R.J., Crystal Plasticity. Journal of Applied Mechanics-Transactions of the Asme, 1983. 50(4B): p. 921-934.

11. YG, H., A user-material subroutine incorporating single crystal plasticity in the ABAQUS finite element program. 1991, Harvard University.

12. Bassani, J.L. and T.Y. Wu, Latent Hardening in Single-Crystals .2. Analytical Characterization and Predictions. Proceedings of the Royal Society of London Series a-Mathematical Physical and Engineering Sciences, 1991. 435(1893): p. 21-41.

13. Lu, C., et al., Crystal plasticity modeling of texture evolution and heterogeneity in equal channel angular pressing of aluminum single crystal. Acta Materialia, 2011. 59(9): p. 35813592.

14. Peirce, D., R.J. Asaro, and A. Needleman, Material Rate Dependence and Localized Deformation in Crystalline Solids. Acta Metallurgica, 1983. 31(12): p. 1951-1976.

15. R, H., Generalized constitutive relations for incremental deformation of metal crystals by multislip. Journal of the Mechanics and Physics of Solids, 1966. 14(2): p. 95-102.

16. Fischer-Cripps, A.C., The IBIS Handbook of Nanoindentation. 2009: Fischer-Cripps Laboratories Pty Ltd.

17. Liu, Y., et al., Combined numerical simulation and nanoindentation for determining mechanical properties of single crystal copper at mesoscale. Journal of the Mechanics and Physics of Solids, 2005. 53(12): p. 2718-2741.

18. Liu, Y., et al., Orientation effects in nanoindentation of single crystal copper. International Journal of Plasticity, 2008. 24(11): p. 1990-2015.

19. Kuo, J.C. and I.H. Huang, Extraction of Plastic Properties of Aluminum Single Crystal Using Berkovich Indentation. Materials Transactions, 2010. 51(11): p. 2104-2108.

20. Franciosi, P., M. Berveiller, and A. Zaoui, Latent Hardening in Copper and Aluminum SingleCrystals. Acta Metallurgica, 1980. 28(3): p. 273-283.

21. Huynh, N.N., et al., A study of microstructural evolution around crack tip using crystal plasticity finite-element method. Proceedings of the Institution of Mechanical Engineers Part J-Journal of Engineering Tribology, 2008. 222(J3): p. 183-192.

22. Liu, Q., et al., Heterogeneous microstructures and microtextures in cube-oriented Al crystals after channel die compression. Metallurgical and Materials Transactions a-Physical Metallurgy and Materials Science, 1998. 29(9): p. 2333-2344. 
23. Akef, A., et al., Recrystallization of High Symmetry Aluminum Single-Crystals after PlaneStrain Compression. Textures and Microstructures, 1991. 14: p. 617-622.

24. Oliver, W.C. and G.M. Pharr, An Improved Technique for Determining Hardness and ElasticModulus Using Load and Displacement Sensing Indentation Experiments. Journal of Materials Research, 1992. 7(6): p. 1564-1583.

25. Simmons, G. and H. Wang, Single Crystal Elastic Constants and Calculated Aggregate Properties: A Handbook. 2nd ed. 1971, Cambridge: The M. I. T. Press.

26. Peralta, P., et al., Characterization of surface deformation around vickers indents in monocrystalline materials. Metallurgical and Materials Transactions a-Physical Metallurgy and Materials Science, 2004. 35A(8): p. 2247-2255.

27. Wert, J.A., Q. Liu, and N. Hansen, Dislocation boundary formation in a cold-rolled cubeoriented Al single crystal. Acta Materialia, 1997. 45(6): p. 2565-2576.

28. Zaafarani, N., et al., On the origin of deformation-induced rotation patterns below nanoindents. Acta Materialia, 2008. 56(1): p. 31-42.

29. Zaafarani, N., et al., Three-dimensional investigation of the texture and microstructure below a nanoindent in a Cu single crystal using 3D EBSD and crystal plasticity finite element simulations. Acta Materialia, 2006. 54(7): p. 1863-1876. 